\title{
Discrete representations of a continuum economy
}

\author{
Carlos Hervés-Beloso ${ }^{1}$, Emma Moreno-García ${ }^{2}$, \\ Carmelo Núñez-Sanz ${ }^{3}$ \\ 1 R.G.E.A., Universidad de Vigo \\ e-mail: cherves@uvigo.es \\ 2 Facultad de Economía y Empresa, Universidad de Salamanca \\ e-mail: emmameusal.es \\ 3 Departamento de Economía, Universidad Carlos III de Madrid \\ e-mail: cnunez@eco.uc3m.es
}

\begin{abstract}
We consider an atomless economy in which the continuum of agents is represented by a real interval. By dividing the interval and associating to every agent in each subinterval the same initial endowments and preferences, we define sequences of discrete economies as approximations to the initial continuum economy. We obtain convergence results for the core (or, alternatively, for the set of Walrasian allocations) of the continuum economy in terms of the cores of the approximating discrete economies. Finally, we state some counterexamples which provide a boundary for more general results in this framework.
\end{abstract}

Mathematics Subject Classification (2000): 91B54

Journal of Economic Literature Classification: D59, D49, D00, D11

\section{Introduction}

Since Aumann (1964) introduced a model of a continuum economy and showed the equivalence between the core and the set of Walrasian allocations, the hypotheses of nonatomicity and perfect competition have been

C. Hervés and E. Moreno acknowledge support by Research Grant BEC2000-1388-C0401 from the Dirección General de Investigación Científica y Técnica (DGICYT), Spanish Ministry of Education.

E. Moreno acknowledges support from the Spanish Ministry of Education through a postdoctoral fellowship in 1997 and from a Training and Mobility of Researchers (E.C.) fellowship in 1998 while visiting Universidade Nova de Lisboa. 
joined. The core equivalence theorem has been refined in many directions, suggesting that there should be a notion of approximation for which the core allocations of large economies approximate core (or Walrasian) allocations of atomless economies. These are important matters for the interpretation of any equivalence theorem, justifying the attention which we give to this asymptotic analysis.

The aim of this paper is to discretize a continuum economy providing a framework to deal with finite approximations of the atomless economy and to present core (or, alternatively, Walrasian equilibrium) convergence results.

A standard way to handle a continuous variable on an interval (for instance, in order to calculate or approximate a solution) is to discretize it; this is to partition the interval, associating to each subinterval a single value. In our case, under the assumption that a finite number of different characteristics will define an approximation to the atomless economy, a precise initial endowment and a precise preference relation then identify a group of agents who are considered identical. The common endowment of agents belonging to the same type must be given by the average real endowment. However, it is not clear what preference relation should be associated to each small subinterval representing the same type of agent. In this paper, we address first the case where the common preference relation is given by an "average" preference and then we consider the "unanimous" preference.

In order to formalize these ideas, given a continuum economy we define, for each $n$, a new continuum economy with a finite number, namely $2^{n}$, of different agent characteristics. To each one of these new continuum economies with a finite number of types of agent, we associate a finite economy with $2^{n}$ agents. We argue that, as $n$ increases (i.e., as the number of different agent characteristics increases or, alternatively, as the number of agents increases), the initial continuum economy is better approximated by the sequence of discrete economies. The main results of this paper are concerned with necessary or sufficient conditions for an allocation to belong to the core of the initial continuum economy, in terms of the sequence of allocations in the core of the associated approximating economies.

A great deal of work has been done on core convergence results, showing that the proper setting for the theoretical study of competition corresponds to large economies. In fact, after Debreu-Scarf's (1963) core convergence theorem, different formalizations and extensions of Edgeworth's (1881) conjecture have been a major focus of the literature in mathematical economics during the 70s and 80s (see Anderson (1992) for a survey). In these papers the theorems state that, for suitable sequences of economies, a measure of the degree of non-competitiveness tends to zero or, alternatively, an "approximate" decentralization of core allocations in large economies is proved. 
Related works on limit properties of the core include Kannai $(1970,1972)$ and Hildenbrand $(1974,1982)$, who obtain continuity properties of the core of a market by considering purely competitive sequences of economies (i.e., sequences of finite economies for which the number of agents converges to infinity and the distribution of agents' characteristics in the market converges to a limit distribution defining an abstract limit economy).

However, we start by considering a continuum economy and then define a sequence of discrete representations approximating the initial atomless economy. Moreover, the sequence of discrete economies we define is not generally a purely competitive sequence of economies converging to the given continuum economy (as showed in the example given in Sect. 4 and Examples 5.2 and 5.3 in Sect. 5). Thus, the paper extends the known core convergence results even if the sequence of discrete economies (obtained from a continuum economy) is not a purely competitive sequence of economies converging to the initial atomless economy.

The paper is organized as follows. In Sect. 2 we present definitions and notation. In Sect. 3 we define sequences of discrete representations of the continuum economy. In Sect. 4 we present an example, which shows that the sequences of discrete representations of a continuum economy do not need to be purely competitive sequences of economies. Therefore, the core convergence results obtained in this paper cannot be regarded as particular cases of the well-known core convergence results for purely competitive sequences of economies. In Sects. 5 and 6 we present the main results of the paper. In Sect. 5 we study the case in which the common preference of all agents of the same type is the average preference whereas in Sect. 6 we address the case in which the common preference is the unanimous preference. In both situations we give examples which provide a boundary for more general results in this framework.

\section{Definitions and notation}

We consider a pure exchange economy $\mathcal{E}_{c}=\left((I, \mathcal{A}, \mu), X_{t}, \omega(t), \succeq_{t}\right.$, $t \in I)$. The commodity space is the Euclidean space $\mathbb{R}^{\ell}$. Let $x=\left(x_{h}\right)_{h=1}^{\ell}$, $y=\left(y_{h}\right)_{h=1}^{\ell} \in \mathbb{R}^{\ell}$. If $x_{h} \geq y_{h}$ for every $h=1, \ldots, \ell$, we write $x \geq y$. If $x_{h}>y_{h}$ for every $h=1, \ldots, \ell$, we write $x \gg y$. For simplicity, we assume that the space of agents is $(I, \mathcal{A}, \mu)$, where $I$ is the real interval $[0,1], \mathcal{A}$ is the Lebesgue $\sigma$-algebra of subsets of $I$, and $\mu$ is the Lebesgue measure. Each agent $t \in I$ is characterized by her consumption set $X_{t}=\mathbb{R}_{+}^{\ell}$, her initial endowment $\omega(t) \in \mathbb{R}_{+}^{\ell}$, and her preference relation $\succeq_{t}$.

We state the following assumptions on endowments and preference relations. 
(H.1) The mapping $\omega: I \rightarrow \mathbb{R}_{+}^{\ell}$, that associates to each agent her initial endowment, is integrable and $\int_{I} \omega(t) d \mu(t) \gg 0$.

An allocation is an integrable function $f: I \rightarrow \mathbb{R}_{+}^{\ell}$. An allocation $f$ is a feasible allocation if $\int_{I} f(t) d \mu(t) \leq \int_{I} \omega(t) d \mu(t)$.

A preference relation $\succeq$ is (weakly) monotone if $x \geq y$ implies that $x \succeq y$; and it is said to be strictly monotone if $x \geq y$ and $x \neq y$ implies that $x \succ y$. The strict monotonicity on preferences was used in Kannai (1970) and Hildenbrand (1974), addressing continuity properties of the core and purely competitive sequences of economies, respectively.

(H.2) The preference relation $\succeq_{t}$ is a complete, continuous and monotone preorder for every agent $t \in I$.

Note that (H.2) implies that each $\succeq_{t}$ is represented by a continuous and monotone utility function $U(t, \cdot): \mathbb{R}_{+}^{\ell} \rightarrow \mathbb{R}$ (see Eilenberg (1941), Debreu (1954) or Debreu (1983), pp. 105-110). We denote by $\mathcal{C}\left(\mathbb{R}_{+}^{\ell}\right)$ the set of all real continuous functions defined on $\mathbb{R}_{+}^{\ell}$. We endow the set $\mathcal{C}\left(\mathbb{R}_{+}^{\ell}\right)$ with the compact-open topology.

(H.3) The mapping $U: I \rightarrow \mathcal{C}\left(\mathbb{R}_{+}^{\ell}\right)$, which associates to each agent $t \in$ $I$ a utility function $U(t, \cdot)$ representing her preference relation, is measurable.

Remark. Note that (H.3) follows from the standard measurability assumption on preferences. To see this, let $\mathcal{P}$ denote the set of continuous preference relations which is a subspace of $\mathcal{C}$, the space of nonempty closed subsets of $\mathbb{R}_{+}^{\ell} \times \mathbb{R}_{+}^{\ell}$. We consider the closed convergence topology on $\mathcal{C}$. The standard assumption is that the mapping $P: I \rightarrow \mathcal{P}$, which associates to each agent her preference relation, is measurable (see Aumann (1964)). Therefore, there exists a continuous function $\mathcal{U}: \mathcal{P} \times \mathbb{R}_{+}^{\ell} \rightarrow \mathbb{R}$ such that $\mathcal{U}(\succeq, \cdot)$ is a utility function which represents the preference relation $\succeq \in \mathcal{P}$ (see Mas-Colell (1977)). Then, the function $\mathcal{V}: \mathcal{P} \rightarrow \mathcal{C}\left(\mathbb{R}_{+}^{\ell}\right)$, given by $\mathcal{V}(\succeq)=\mathcal{U}(\succeq, \cdot)$, is a continuous function (see Wilansky (1983), Theorem 13.3.4). Therefore, the composition of $P$ with $\mathcal{V}, U: I \rightarrow \mathcal{C}\left(\mathbb{R}_{+}^{\ell}\right)$, which associates to each agent $t \in I$ an utility function $U_{t} \in \mathcal{C}\left(\mathbb{R}_{+}^{\ell}\right)$ representing her preference relation, is measurable. Thus, by (H.2), Assumption (H.3) is equivalent to the usual assumption requiring the measurability of the mapping that associates to each agent her preference relation.

We recall that a coalition of agents is any positive measure subset of the set of agents. A feasible allocation belongs to the core of the economy if it is not blocked by any coalition of agents. A coalition $S$ blocks an allocation $f$ via another allocation $g$ in the economy $\mathcal{E}_{c}$ if 
(i) $\int_{S} g(t) d \mu(t) \leq \int_{S} \omega(t) d \mu(t)$, and

(ii) $g(t) \succ_{t} f(t)$ for almost all $t \in S$.

We denote by $\operatorname{Core}\left(\mathcal{E}_{c}\right)$ the set of allocations which belong to the core of the economy $\mathcal{E}_{c}$. Given a family $\mathcal{F}$ of coalitions, we say that an allocation $f$ belongs to the $\mathcal{F}$-Core of the economy $\mathcal{E}_{c}$, and we write $f \in \mathcal{F}$-Core $\left(\mathcal{E}_{c}\right)$ if it is not blocked by any coalition $S \in \mathcal{F}$.

On the other hand, given $a, b \in \mathbb{R}^{\ell}$ let $a \ominus b$ be the vector in $\mathbb{R}_{+}^{\ell}$ whose $k$ th coordinate is $\max \left\{a_{k}-b_{k}, 0\right\}$. Given $\varepsilon>0$, as in Kannai (1970), we say that an allocation $f$ belongs to the $\varepsilon$-Core of the economy $\mathcal{E}_{c}$, and we write $f \in \varepsilon$-Core $\left(\mathcal{E}_{c}\right)$ if $g(t) \succ_{t} f(t)$ for almost all $t \in S$ implies that the inequality $\int_{S} g(t) d \mu(t)<\int_{S} \omega(t) d \mu(t) \ominus \varepsilon$ does not hold.

\section{Sequences of discrete approximations}

As we noted in the introduction, our aim is to analyze discrete approximations to a given economy with a continuum of agents represented by the real interval $[0,1]$. We observe that a standard way to deal with a continuous variable on an interval is to make it discrete by dividing the interval into subintervals and associating to each subinterval a single value.

In our case, given a continuum economy $\mathcal{E}_{c}$, for each positive integer $n$ we define a continuum economy $\mathcal{E}_{c}^{n}$ with only a finite number of different agent characteristics. Specifically, we assume that the set of agents $I$ is divided into $2^{n}$ pairwise disjoint subintervals, each representing a type of agent. That is, $I=\bigcup_{i=1}^{2^{n}} I_{i}^{n}$, where $I_{i}^{n}=\left[\frac{i-1}{2^{n}}, \frac{i}{2^{n}}\right)$, if $i \neq 2^{n}$, and $I_{2^{n}}^{n}=\left[\frac{2^{n}-1}{2^{n}}, 1\right]$. We refer to $I_{i}^{n}$ as the set of agents of type $i$ in the economy $\mathcal{E}_{c}^{n}$. In this economy, the consumption set is $\mathbb{R}_{+}^{\ell}$, and the common preference relation and initial endowment for all the agents of type $i$ are denoted $\succeq_{i}^{n}$ and $\omega_{i}^{n}$, respectively.

Let $\mathcal{E}_{n}$ be the discrete economy associated with the continuum economy $\mathcal{E}_{c}^{n}$, that is, $\mathcal{E}_{n}$ is an economy with $2^{n}$ agents. Each agent $i \in\left\{1, \ldots, 2^{n}\right\}$ is characterized by her initial endowment $\omega_{i}^{n}$ and her preference relation $\succeq_{i}^{n}$.

Observe that an allocation $f$ in our economy $\mathcal{E}_{c}$ can be interpreted either as an allocation $f^{n}$ in $\mathcal{E}_{c}^{n}$, or as an allocation $x^{n}=\left(x_{1}, \ldots, x_{2^{n}}\right)$ in $\mathcal{E}_{n}$, where $x_{i}^{n}=f^{n}(t)=\frac{1}{\mu\left(I_{i}^{n}\right)} \int_{I_{i}^{n}} f(t) d \mu(t)$ for all $t \in I_{i}^{n}$. Reciprocally, an allocation $x^{n}$ in $\mathcal{E}_{n}$ can be interpreted as an allocation $f$ in $\mathcal{E}_{c}^{n}$ or in $\mathcal{E}_{c}$, where $f$ is the step function defined by $f(t)=x_{i}$ if $t \in I_{i}^{n}$. In particular, the initial endowment for the agents of type $i$ in the economy $\mathcal{E}_{c}^{n}$ (or for agent $i$ in $\mathcal{E}_{n}$ ) is $\omega_{i}^{n}=\frac{1}{\mu\left(I_{i}^{n}\right)} \int_{I_{i}^{n}} \omega(t) d \mu(t)$. 
With $x^{n}$ and $f^{n}$ as above, it is easy to prove that, if $x^{n}$ is blocked by a coalition $S$ in the economy $\mathcal{E}_{n}$, then $f^{n}$ is blocked by the coalition $S_{c}=\bigcup_{i \in S} I_{i}^{n}$ in the economy $\mathcal{E}_{c}^{n}$. Moreover, it is straightforward to show that, under convexity of preferences, $x^{n} \in \operatorname{Core}\left(\mathcal{E}_{n}\right)$ iff $f^{n} \in \mathcal{F}^{n}$ - $\operatorname{Core}\left(\mathcal{E}_{c}^{n}\right)$, where $\mathcal{F}^{n}$ denotes the $\sigma$-algebra generated by the subintervals $I_{i}^{n}, i=1, \ldots, 2^{n}$.

For preferences we consider two different scenarios. In Sect. 5, we assume that, for each $n$, the preference relation $\succeq_{i}^{n}$ of agents of type $n$ is represented by the utility function $U^{n}(t, x)=\frac{1}{\mu\left(I_{i}^{n}\right)} \int_{I_{i}^{n}} U(t, x) d \mu(t)$ for each agent $t \in I_{i}^{n}$. In this case, we refer to $\succeq_{i}^{n}$ as the average preference. We denote the approximations $\mathcal{E}_{c}^{n}$ and $\mathcal{E}_{n}$ to $\mathcal{E}_{c}$ by $\overline{\mathcal{E}}_{c}^{n}$ and $\overline{\mathcal{E}}_{n}$, respectively, when we consider this average preference. Observe that, if $U(t, \cdot)$ is continuous (resp., monotone, strictly monotone, concave) for almost all $t \in I$, then $U^{n}(t, \cdot)$ is also continuous (resp., monotone, strictly monotone, concave) for every $t \in I$ and for every $n$.

Later, in Sect. 6, we assume the unanimous preference relation. Then, for each $n$ the preference relations are defined as follows:

$$
x \succeq_{i}^{n} y \Leftrightarrow x \succeq_{t} y \text { for almost all } t \in I_{i}^{n} .
$$

In this case, we refer to $\succeq_{i}^{n}$ as the unanimous preference. This preference states that a consumption vector is preferred to another if, in the economy $\mathcal{E}_{c}$, it is preferred by almost all the agents belonging to the subinterval $I_{i}^{n}$. When we consider the unanimous preference, we denote the approximations $\mathcal{E}_{c}^{n}$ and $\mathcal{E}_{n}$ to the atomless economy $\mathcal{E}_{c}$ by $\hat{\mathcal{E}}_{c}^{n}$ and $\hat{\mathcal{E}}_{n}$, respectively. Observe that, if $\succeq_{t}$ is continuous (resp., monotone, strictly monotone, convex, strictly convex) for almost all $t \in I$, then $\succeq_{i}^{n}$ is continuous (resp., monotone, strictly monotone, convex, strictly convex) for every $i$ and for all $n$. Observe also that the unanimous preference relation is transitive but, in general, it is not complete.

\section{An example}

We consider a continuum economy $\mathcal{E}_{c}$ with a single commodity and a continuum of agents represented by the real interval $I=[0,1]$, endowed with the Lebesgue measure $\mu$. We can construct a Cantor-like set $K \subset I$ such that $0<\mu(A \bigcap K)<\mu(A)$ for every open subinterval $A$ of the set of agents $I$. 
For each agent $t \in I=[0,1]$, let $\succeq_{t}$ be her preference relation, which is represented by the utility function $U(t, \cdot)$, given by

$$
U(t, x)=\left\{\begin{array}{l}
x \text { if } x \leq 1, \\
1 \text { if } x \geq 1 \text { and } t \in K, \\
x \text { if } x \geq 1 \text { and } t \notin K .
\end{array}\right.
$$

We denote by $\mathcal{C}\left(\mathbb{R}_{+}\right)$the set of all real continuous functions defined on $\mathbb{R}_{+}$. Note that the function $U: I \rightarrow \mathcal{C}\left(\mathbb{R}_{+}\right)$, which associates to each agent $t \in I$ her utility function $U(t, \cdot)$, is measurable.

We remark that any subinterval of the set of agents $I$ contains a positive measure set of agents belonging to the Cantor-like set $K$ and a positive measure set of agents belonging to $I \backslash K$. Thus, if $y<x$ and $y<1$, then every agent prefers $x$ rather than $y$; and, if $1 \leq y<x$, every subinterval includes a positive measure set of agents who prefer $x$ rather than $y$, and a positive measure set of agents who are indifferent between $x$ and $y$. Therefore, for each $n$, both the average preference and the unanimous preference are represented by the same utility function $U^{n}(t, x)=x$ for all agents. Thus, this sequence of preference relations is strictly monotone and does not converge (in any reasonable topology) to the preference relations which define the initial continuum economy $\mathcal{E}_{c}$.

We recall that a sequence of finite economies is a purely competitive sequence of economies if the number of agents tends to infinity, the sequence of preference-endowment distribution $v_{n}$ converges weakly to a limit preference-endowment distribution $v$, the sequence of mean endowments converges to the mean endowment given by the limit distribution and this limit mean endowment is strictly positive (see Kannai (1970) or Hildenbrand $(1974,1982))$.

Thus, the example proves that the sequences $\overline{\mathcal{E}}_{n}$ and $\hat{\mathcal{E}}_{n}$ of discrete economies obtained from $\mathcal{E}_{c}$ do not result in purely competitive sequences of economies converging to the limit economy $\mathcal{E}_{c}$. Moreover, since the Cantorlike set $K$ may have any measure between 0 and 1 , the same conclusions hold for any voting rule which may define an "aggregate" preference for the sequence of finite economies. Therefore, the core convergence results stated in this paper cannot be considered as particular cases of the well-known convergence results addressing purely competitive sequences of economies.

\section{Average preference}

In this section, we consider sequences $\overline{\mathcal{E}}_{c}^{n}$ and $\overline{\mathcal{E}}_{n}$ of discrete economies defined by the average preference. We remark that most of the assumptions required in this paper are the standard ones for continuum economies: see, for instance, Aumann $(1964,1966)$ or Mas-Colell (1977), where a selection 
of utility functions satisfies Assumption (H.3). Observe that the average preference relation, as defined in Sect. 3, may depend on the criterion for the selection of the utility functions representing the agents' preference relations in the continuum economy $\mathcal{E}_{c}$. However, we remark that the results we obtain do not depend on the utility functions chosen for representing the agents' preferences in the continuum economy.

\subsection{Main results}

In this section we prove two main results. The first states necessary conditions for an allocation $f$ to belong to the core of the economy $\mathcal{E}_{c}$, in terms of the core of its approximating economies $\overline{\mathcal{E}}_{n}$ and $\overline{\mathcal{E}}_{c}^{n}$. The second is a weaker converse.

Next we state the main result in this section.

Theorem 5.1. Let $\mathcal{E}_{c}$ be a continuum economy under Assumptions (H.1), $(H .2)$ and (H.3). Let $f$ be an allocation in $\mathcal{E}_{c}$. The approximation $\overline{\mathcal{E}}_{n}$ (resp., $\left.\overline{\mathcal{E}}_{c}^{n}\right)$ to $\mathcal{E}_{c}$ satisfies the following property:

if $x^{n} \in \operatorname{Core}\left(\overline{\mathcal{E}}_{n}\right)$ (resp., $f^{n} \in \operatorname{Core}\left(\overline{\mathcal{E}}_{c}^{n}\right)$ ) for all $n \geq n_{0}$, then $f \in$ $\operatorname{Core}\left(\mathcal{E}_{c}\right)$.

Remark. Indeed we can see in the proof of the theorem that, if an allocation $f$ does not belong to the core of the initial continuum economy $\mathcal{E}_{c}$, then the corresponding allocation $x^{n}$ does not belong to the core of the finite economy $\overline{\mathcal{E}}_{n}$ for all large enough $n$. Therefore, we can conclude that, if $f^{n} \in \mathcal{F}^{n}$-Core $\left(\overline{\mathcal{E}}_{c}^{n}\right)$ for all $n \geq n_{0}$, then $f \in \operatorname{Core}\left(\mathcal{E}_{c}\right)$.

The corresponding converse of Theorem 5.1 does not hold, as we show in Sect. 5.2. However, in the case of strictly monotone preference relations, we can obtain a weaker converse result. For this, we state the following assumption.

(H.2) $)^{\prime}$ The preference relation $\succeq_{t}$ is a complete, continuous and strictly monotone preorder for every agent $t \in I$.

Let $\hat{\mathcal{P}}$ be the set of all strictly monotone and continuous preorders defined on $\mathbb{R}_{+}^{\ell}$. Each element $\succeq \in \hat{\mathcal{P}}$ can be represented by a unique continuous utility function $U$ such that $U(x)=\|x\|$ if $x$ belongs to the principal diagonal (see Kannai (1970)). Let $\hat{\mathcal{U}}$ denote the set of all the utility functions obtained as above. As in Kannai (1970), we consider on $\hat{\mathcal{P}}$ the metric $\rho$ given by

$$
\rho\left(\succeq_{1}, \succeq_{2}\right)=\max _{x \in \mathbb{R}_{+}^{\ell}} \frac{\left|U_{1}(x)-U_{2}(x)\right|}{1+\|x\|^{2}}
$$

where $U_{i}$ belongs to $\hat{\mathcal{U}}$ and represents $\succeq_{i}$. 
Next, we obtain a weaker converse version of Theorem 5.1 in terms of the $\varepsilon$-Core concept.

Theorem 5.2. Let $\mathcal{E}_{c}$ be a continuum economy under Assumptions (H.1), $(H .2)^{\prime}$ and $(H .3)$. Let $U(t, \cdot) \in \hat{\mathcal{U}}$ for almost all $t \in I$. If $f \in \operatorname{Core}\left(\mathcal{E}_{c}\right)$ then, for each $\varepsilon>0$, there exists $n_{0}$ such that, for all $n \geq n_{0}$, $f^{n}$ belongs to the $\varepsilon$-Core of $\overline{\mathcal{E}}_{c}^{n}$.

\subsection{Some counterexamples}

Example 5.1. This example shows that the underlying converse result of Theorem 5.1 is not true. Precisely, we define an economy $\mathcal{E}_{c}$ such that there exists $f \in \operatorname{Core}\left(\mathcal{E}_{c}\right)$, but $f^{n} \notin \operatorname{Core}\left(\overline{\mathcal{E}}_{c}^{n}\right)$ for all $n$.

By Cantor's nested intervals theorem we can take $\alpha_{1}, \alpha_{2}, \beta_{1}, \beta_{2}$, with $0<\alpha_{1}<\beta_{1}<\alpha_{2}<\beta_{2}$, such that:

(i) for all odd $n$ and for each $i=i(n), j=j(n) \in\left\{1, \ldots, 2^{n}\right\}$, with $i \neq j$, such that $\alpha_{1} \in I_{i}^{n}$ and $\alpha_{2} \in I_{j}^{n}$, we have $\mu\left(\left\{t \in I_{i}^{n} \mid t<\alpha_{1}\right\}\right)>$ $\mu\left(\left\{t \in I_{i}^{n} \mid t>\alpha_{1}\right\}\right)$ and $\mu\left(\left\{t \in I_{j}^{n} \mid t<\alpha_{2}\right\}\right)<\mu\left(\left\{t \in I_{j}^{n} \mid t>\alpha_{2}\right\}\right)$, and

(ii) for all even $n$ and for each $h=h(n), k=k(n) \in\left\{1, \ldots, 2^{n}\right\}$, with $h \neq k$, such that $\beta_{1} \in I_{h}^{n}$ and $\beta_{2} \in I_{k}^{n}$, we have $\mu\left(\left\{t \in I_{h}^{n} \mid t<\beta_{1}\right\}\right)<$ $\mu\left(\left\{t \in I_{h}^{n} \mid t>\beta_{1}\right\}\right)$ and $\mu\left(\left\{t \in I_{k}^{n} \mid t<\beta_{2}\right\}\right)>\mu\left(\left\{t \in I_{k}^{n} \mid t>\beta_{2}\right\}\right)$.

Let the economy $\mathcal{E}_{c}$ have $\mathbb{R}^{2}$ as commodity space. Each agent $t \in[0,1]$ is characterized by her initial endowment $\omega(t)$ and her utility function $U(t,(x, y))$, given by

$$
\begin{gathered}
\omega(t)=\left\{\begin{array}{l}
(1,0) \text { if } t \in\left[0, \alpha_{1}\right) \cup\left[\beta_{1}, \alpha_{2}\right) \cup\left[\beta_{2}, 1\right], \\
(0,1) \text { if } t \in\left[\alpha_{1}, \beta_{1}\right) \bigcup\left[\alpha_{2}, \beta_{2}\right),
\end{array}\right. \\
U(t,(x, y))=\left\{\begin{array}{l}
2 x+y \text { if } t \in\left[0, \alpha_{1}\right) \bigcup\left[\beta_{1}, \alpha_{2}\right) \bigcup\left[\beta_{2}, 1\right], \\
x+2 y \text { if } t \in\left[\alpha_{1}, \beta_{1}\right) \bigcup\left[\alpha_{2}, \beta_{2}\right) .
\end{array}\right.
\end{gathered}
$$

In the economy $\overline{\mathcal{E}}_{c}^{2 n+1}$, though all agents of type $i_{2 n+1}$ prefer the first commodity, all agents of type $j_{2 n+1}$ prefer the second one. On the other hand, in the economy $\overline{\mathcal{E}}_{c}^{2 n}$ while all agents of type $h_{2 n}$ prefer the first commodity, all the agents of type $k_{2 n}$ prefer the second one. It is not hard to show that the allocation $f$, given by $f(t)=\omega(t)$, belongs to Core $\left(\mathcal{E}_{c}\right)$. However, $f^{n} \notin$ Core $\left(\overline{\mathcal{E}}_{c}^{n}\right)$ for all $n$. In fact, for each $n$ the coalition $S_{2 n+1}=I_{i}^{2 n+1} \cup I_{j}^{2 n+1}$ blocks the allocation $f^{2 n+1}$ via $g_{2 n+1}$ in the economy $\mathcal{E}_{c}^{2 n+1}$, and the coalition $S_{2 n}=I_{h}^{2 n} \bigcup I_{k}^{2 n}$ blocks $f^{2 n}$ via $g_{2 n}$ in the economy $\mathcal{E}_{c}^{2 n+1}$. The allocations 
$g_{n}$ are defined as follows:

$$
\begin{gathered}
g_{2 n+1}(t)=\left\{\begin{array}{l}
f^{2 n+1}(t)+\left(\varepsilon_{2 n+1},-\varepsilon_{2 n+1}\right) \text { if } t \in I_{i_{2 n+1}}^{2 n+1}, \\
f^{2 n+1}(t)+\left(-\varepsilon_{2 n+1}, \varepsilon_{2 n+1}\right) \text { if } t \in I_{j_{2 n+1}}^{2 n+1},
\end{array}\right. \\
g_{2 n}(t)=\left\{\begin{array}{l}
f^{2 n}(t)+\left(\varepsilon_{2 n},-\varepsilon_{2 n}\right) \text { if } t \in I_{h_{2 n}}^{2 n}, \\
f^{2 n}(t)+\left(-\varepsilon_{2 n}, \varepsilon_{2 n}\right) \text { if } t \in I_{k_{2 n+1}}^{2 n},
\end{array}\right.
\end{gathered}
$$

where $\varepsilon_{n}$ is any positive real number such that $g_{n}(t)>0$.

Observe that, although the functions $U$ and $\omega$ are piecewise continuous, they can be chosen to be continuous, leading us to the same claim.

Remark. The example in Sect. 4 shows that the proof of Theorem 5.2 is not valid if the strict monotonicity assumed on preferences $\succeq_{t}$ is deleted. In particular, the sequence of average preferences in that example converges, in the Kannai sense of the metric $\rho$, to strictly monotone preference relations, which, therefore, differ from the preferences $\succeq_{t}$ considered in the initial continuum economy. Therefore, Lemma 5 (in the Appendix) does not hold if $\succeq_{t}$ are not strictly monotone.

\section{Unanimous preference}

\subsection{Main results}

Our aim is to obtain sufficient conditions and necessary conditions for an allocation $f$ to belong to the core of the economy $\mathcal{E}_{c}$, in terms of the cores of the economies $\hat{\mathcal{E}}_{c}^{n}$ (or $\hat{\mathcal{E}}_{n}$ ) defined by the unanimous preference relation. For this, we state the following continuity assumption.

(H.4) The mapping $U: I \rightarrow \mathcal{C}\left(\mathbb{R}_{+}^{\ell}\right)$, which associates to each agent $t \in$ $I$ a utility function $U(t, \cdot)$ representing her preference relation, is continuous with respect to the compact-open topology.

The standard assumption on preferences (see Aumann $(1964,1966)$ ) requires the mapping which associates to each agent her preference relation to be measurable. As we remark after stating Assumption (H.3) this means that the mapping $U$ is measurable. Measurability of $U$ implies that, for $\epsilon>0$, the function $U$ is continuous on a compact subset $K \subset I$, with $\mu(K)>1-\epsilon$. Thus, Assumption (H.4) is stronger than (H.3) and requires continuity of $U$ on $I$ and not only on compact sets with measure $1-\epsilon$ for any $\epsilon>0$.

We will show that Assumption (H.4) is a necessary condition for the next theorem (see Example 6.3 in the next subsection). 
Theorem 6.1. Let $\mathcal{E}_{c}$ be a continuum economy under Assumptions (H.1), $(H .2)^{\prime}$ and (H.4). Let $f$ be a continuous allocation in $\mathcal{E}_{c}$. Then the approximation $\hat{\mathcal{E}}_{c}^{n}$ to $\mathcal{E}_{c}$ satisfies the following property:

if $f^{n} \in \operatorname{Core}\left(\hat{\mathcal{E}}_{c}^{n}\right)$ for all $n \geq n_{0}$, then $f \in \operatorname{Core}\left(\mathcal{E}_{c}\right)$.

Remark. In fact we prove that, if an allocation $f$ is blocked by the coalition $S$ via $g$ in the economy $\mathcal{E}_{c}$, then $f^{n}$ is also blocked by the same coalition $S$ and via the same allocation $g$ in the economy $\hat{\mathcal{E}}_{c}^{n}$. Moreover, let $f$ be a piecewise continuous function with simple discontinuities at a finite subset of $\mathbb{D}=\left\{i 2^{-n} \mid i=0, \ldots, 2^{n} ; n \in \mathbb{N}\right\}$. The proof above shows that, if $f^{n} \in \operatorname{Core}\left(\hat{\mathcal{E}}_{c}^{n}\right)$ for all $n \geq \bar{n}$, then $f \in \operatorname{Core}\left(\mathcal{E}_{c}\right)$.

In order to find necessary conditions for an allocation $f$ to belong to the core of the economy $\mathcal{E}_{c}$, preference relations are also required to be convex:

(H.5) The preference relation $\succeq_{t}$ is convex for almost all the agents $t \in I$.

We recall that a preference relation $\succeq$ is said to be convex if $y \succeq x$ implies that $\lambda y+(1-\lambda) x \succeq x$ for any $\lambda, 0 \leq \lambda \leq 1$. This is equivalent to assuming that, if $y \succ x$ and $z \succ x$, then $\lambda y+(1-\lambda) z \succ x$ for any $\lambda$, $0 \leq \lambda \leq 1$ (see Debreu (1959)). Thus, as we deal with preference relations which are representable by utility functions, (H.5) is equivalent to assuming quasi-concavity of the corresponding utility functions.

The next theorem is a partial converse of Theorem 6.1. First, we recall that $\mathcal{F}^{n}$ denotes the $\sigma$-algebra generated by the subintervals $I_{i}^{n}, i=1, \ldots, 2^{n}$. Now, we define $\mathcal{S}^{n}=\left\{f: I \rightarrow \mathbb{R}_{+}^{\ell} \mid f(t)=f_{i}\right.$ for all $t \in I_{i}^{n}, i=$ $\left.1, \ldots, 2^{n}\right\}$. In other words, $\mathcal{S}^{n}$ is the set of all functions which are $\mathcal{F}^{n}$ measurable.

Theorem 6.2. Let $\mathcal{E}_{c}$ be a continuum economy under Assumptions (H.1) and (H.5). Let $f \in \mathcal{S}^{\bar{n}}$. If $f \in \operatorname{Core}\left(\mathcal{E}_{c}\right)$, then $f \in \mathcal{F}^{n}$-Core $\left(\hat{\mathcal{E}}_{c}^{n}\right)$ for all $n \geq \bar{n}$.

\subsection{Some counterexamples}

Example 6.1. This example is similar to Example 5.1 and shows that the converse of Theorem 6.1 is not true. Precisely, we define an economy $\mathcal{E}_{c}$ such that there exists $f \in \operatorname{Core}\left(\mathcal{E}_{c}\right)$, but $f^{n} \notin \operatorname{Core}\left(\hat{\mathcal{E}}_{c}^{n}\right)$ for all $n$.

Let $\alpha \in I$ be such that, for all odd $n$ and for all $i=i(n)$ with $\alpha \in I_{i}^{n}$, we have $a_{i}^{n}<\alpha<b_{i}^{n}$, where $a_{i}^{n}=\frac{2}{3}\left(\frac{i-1}{2^{n}}\right)+\frac{1}{3}\left(\frac{i}{2^{n}}\right)$ and $b_{i}^{n}=\frac{1}{3}\left(\frac{i-1}{2^{n}}\right)+$ $\frac{2}{3}\left(\frac{i}{2^{n}}\right)$. This implies that $\mu\left(\left\{t \in I_{i}^{n} \mid t<\alpha\right\}\right)<2 \mu\left(\left\{t \in I_{i}^{n} \mid t>\alpha\right\}\right)$ and $\mu\left(\left\{t \in I_{i}^{n} \mid t>\alpha\right\}\right)<2 \mu\left(\left\{t \in I_{i}^{n} \mid t<\alpha\right\}\right)$ for all odd $n$.

In the same way, by Cantor's nested intervals theorem, we choose $\beta$ such that, for all even $n, \mu\left(\left\{t \in I_{i}^{n} \mid t<\beta\right\}\right)<2 \mu\left(\left\{t \in I_{i}^{n} \mid t>\beta\right\}\right)$ and $\mu\left(\left\{t \in I_{i}^{n} \mid t>\beta\right\}\right)<2 \mu\left(\left\{t \in I_{i}^{n} \mid t<\beta\right\}\right)$. 
We consider the economy $\mathcal{E}_{c}$ with the commodity space $\mathbb{R}^{2}$. Each agent $t \in[0,1]$ is characterized by her initial endowment $\omega(t)$ and her preference relation represented by the utility function $U(t,(x, y))$, defined as:

$$
\begin{aligned}
\omega(t) & =\left\{\begin{array}{l}
(1,0) \text { if } t<\alpha \text { or } t>\beta, \\
(0,1) \text { if } \alpha<t<\beta,
\end{array}\right. \\
U(t,(x, y)) & =\left\{\begin{array}{l}
2 x+y \text { if } t<\alpha \text { or } t>\beta, \\
x+2 y \text { if } \alpha<t<\beta .
\end{array}\right.
\end{aligned}
$$

As in Example 5.1, it is clear that the allocation $f$, given by $f(t)=\omega(t)$, belongs to $\operatorname{Core}\left(\mathcal{E}_{c}\right)$. However, if $n$ is an odd number (resp., $n$ is even), then the coalition $S_{n}=I_{i}^{n}$, with $\alpha \in I_{i}^{n}$ (resp., with $\beta \in I_{i}^{n}$ ), blocks the allocation $f^{n}$ via $g_{n}$ in the economy $\hat{\mathcal{E}}_{c}^{n}$, where $g_{n}(t)=f(t)$ for all $t$. To see this, observe that if, for example, $t \in S_{n}$ with $n$ odd, then

$$
U\left(t, f^{n}(t)\right)=\left\{\begin{array}{l}
2\left(\alpha-\frac{i-1}{2^{n}}\right) 2^{n}+\left(\frac{i}{2^{n}}-\alpha\right) 2^{n} \text { if } t<\alpha, \\
\left(\alpha-\frac{i-1}{2^{n}}\right) 2^{n}+2\left(\frac{i}{2^{n}}-\alpha\right) 2^{n} \text { if } t>\alpha .
\end{array}\right.
$$

Therefore, if $t \in S_{n}$, then $f(t)$ is unanimously preferred to $f^{n}(t)$, because $U\left(t, f^{n}(t)\right)<U(t, f(t))$ for all $t \in S_{n}$, since $\max \left\{\alpha-\frac{i-1}{2^{n}}, \frac{i}{2^{n}}-\alpha\right\}<$ $\frac{2^{-n+1}}{3}$.

The case $n$ even is much the same.

Example 6.2. This example shows that the continuity of $f$ in Theorem 6.1 cannot be dropped. To prove this, we consider a continuum economy $\mathcal{E}_{c}$ with two commodities, and let $K$ be a Cantor subset of $I$, with $\mu(K)>0, K=$ $K_{1} \bigcup K_{2}$, with $\mu\left(K_{1}\right)=\mu\left(K_{2}\right), K_{1} \cap K_{2}=\emptyset$, and $I \backslash K=\bigcup_{k=1}^{\infty}\left(a_{k}, b_{k}\right)$. For each agent $t \in I$, let $U(t,(x, y))=(1+x)^{1+\beta(t)}(1+y)^{1-\beta(t)}$, where $\beta: I \rightarrow \mathbb{R}$ is the continuous function defined as

$$
\beta(t)=\left\{\begin{array}{cl}
0 & \text { if } t \in K, \\
\left(t-a_{k}\right)\left(t-b_{k}\right) \sin \left(\frac{1}{\left(t-a_{k}\right)\left(t-b_{k}\right)}\right) & \text { if } t \in\left(a_{k}, b_{k}\right) .
\end{array}\right.
$$

Let $A=\{t \in I \mid \beta(t)>0\}$ and $B=\{t \in I \mid \beta(t)<0\}$.

The initial endowments are given by

$$
\omega(t)=\left\{\begin{array}{cl}
(1+\gamma, 1-\gamma) & \text { if } t \in K_{1}, \\
(1-\gamma, 1+\gamma) & \text { if } t \in K_{2}, \\
(1,0) & \text { if } t \in A, \\
(0,1) & \text { if } t \in B .
\end{array}\right.
$$

It is easy to prove that, if $K \subset S$, then the coalition $S$ blocks the allocation $f=\omega$ via the allocation $g$, defined as $g(t)=(1,1)$ if $t \in K$, and $g(t)=$ $f(t)$ if $t \in S \backslash K$. 
However, $f^{n} \in \operatorname{Core}\left(\hat{\mathcal{E}}_{c}^{n}\right)$ for all $n$. To prove this, let us assume that there exist a coalition $\hat{S}$ and an allocation $g$ such that $f^{n}(t)=\left(f_{1}^{n}(t), f_{2}^{n}(t)\right) \prec_{t}^{n}$ $g(t)=\left(g_{1}(t), g_{2}(t)\right)$. Then, $g_{1}(t)+g_{2}(t)>f_{1}^{n}(t)+f_{2}^{n}(t)$ for all $t \in \hat{S}$, which is a contradiction.

To see the last inequality observe that we have three possibilites:

(a) $I_{i}^{n} \subset A$;

(b) $I_{i}^{n} \subset B$;

(c) $I_{i}^{n} \cap A \neq \emptyset$ and $I_{i}^{n} \bigcap B \neq \emptyset$.

Consider $i$ such that $\mu\left(\hat{S} \cap I_{i}^{n}\right)>0$. If (a) holds, then the agents of type $i$ prefer commodity 1 to commodity 2 , but they do not have commodity 2 and the inequality follows. Case (b) is similar, and finally, if (c) holds, the inequality follows because the agents in $A$ (resp., in $B$ ) prefer commodity 1 (resp., 2) to commodity 2 (resp., 1).

Example 6.3. Now we give an example which shows that, even if the allocation $f$ is a constant function, Theorem 6.1 does not hold if assumption (H.4) is dropped.

For this, let $A, B$ be disjoint subsets of $I$ such that $\mu\left(I_{i}^{n} \bigcap A\right)>0$ and $\mu\left(I_{i}^{n} \cap B\right)>0$, for all $n$ and $i$. For example, we can take $A$ and $B$ as

$$
A=\bigcup_{n=1}^{\infty} \bigcup_{i=1}^{2^{n}} A_{i}^{n}, \quad B=\bigcup_{n=1}^{\infty} \bigcup_{i=1}^{2^{n}} B_{i}^{n}
$$

such that $A_{i}^{n}, B_{i}^{n}$ are non-negligible Cantor subsets of $I_{i}^{n}$, satisfying

$$
\left(\bigcup_{n=1}^{N} \bigcup_{i=1}^{2^{n}} A_{i}^{n}\right) \bigcap\left(\bigcup_{n=1}^{N} \bigcup_{i=1}^{2^{n}} B_{i}^{n}\right)=\emptyset \text { for all } N \text {. }
$$

Consider an economy $\mathcal{E}_{c}$ with two commodities. Each agent $t \in I$ is characterized by her endowment $\omega(t)=(1,1)$, and her utility function, given by

$$
U(t,(x, y))=\left\{\begin{aligned}
2 x+y & \text { if } t \in A \\
x+2 y & \text { if } t \in B, \\
V(t,(x, y)) & \text { if } t \in I \backslash(A \cup B) .
\end{aligned}\right.
$$

The preferences of agents outside the set $A \cup B$ are not relevant in this example. We remark that, by construction of the sets $\mathrm{A}$ and $\mathrm{B}$, the function $U$ is not continuous.

It is clear that the coalition $S=A \cup B$ blocks the allocation $f=\omega$. However, $f^{n} \in \operatorname{Core}\left(\hat{\mathcal{E}}_{c}^{n}\right)$ for all $n$. This is because, if $g(t)=\left(g_{1}(t), g_{2}(t)\right)$ is unanimously preferred to $(1,1)$ by all $t \in S$ with $\mu(S)>0$, then $g_{1}(t)+$ $g_{2}(t)>2$ for all $t \in S$. 
Example 6.4. Lastly, we give an example which shows that, for the case of unanimous preference, an analog to Theorem 5.2 fails if the preferences of the agents are monotone but not strictly monotone. For this, we show that the fact that an allocation $f$ belongs to $\operatorname{Core}\left(\mathcal{E}_{c}\right)$ does not imply that, for each $\varepsilon>0, f^{n}$ belongs to $\varepsilon$-Core $\left(\hat{\mathcal{E}}_{c}^{n}\right)$ for all large enough $n$.

Consider a continuum economy $\mathcal{E}_{c}$ with a single commodity. Each agent $t \in I=[0,1]$ has as initial endowment $\omega(t) \in \mathbb{R}_{+}$, such that $\int_{I} \omega(t) d \mu(t)=$ 1 and $\int_{0}^{\frac{1}{2}} \omega(t) d \mu(t)=2^{-1}+\alpha, 0<\alpha \leq 2^{-1}$. The preference relation of the agent $t \in I$ is represented by the utility function $U(t, \cdot)$, defined as

$$
U(t, x)=\left\{\begin{array}{cl}
x & \text { if } x \leq 2^{-1}+t \\
2^{-1}+t & \text { if } x>2^{-1}+t
\end{array}\right.
$$

It is easy to prove that the allocation $f$, given by $f(t)=2^{-1}+t$, belongs to the core of the economy $\mathcal{E}_{c}$. This is because any agent $t$ is satiated with $f(t)$. However, if $\varepsilon<\alpha$, then $f^{n} \notin \varepsilon$-Core $\left(\hat{\mathcal{E}}_{c}^{n}\right)$ for all $n$. In fact, we claim that, for every $n, f^{n}$ is blocked by the coalition $S=\left[0,2^{-1}\right)$ via $g(t)=1$ for every $t \in S$. To prove our point, let $t_{i}^{n}$ be the midpoint of the subinterval $I_{i}^{n}$. Then for all $I_{i}^{n} \subset S, g(t)=1$ is strictly preferred to $f^{n}(t)=2^{-1}+t_{i}^{n}$ for every agent $t \in\left(t_{i}^{n}, \frac{i}{2^{n}}\right)$, and is indifferent for all the agents $t \in\left[\frac{i-1}{2^{n}}, t_{i}^{n}\right]$.

\section{Appendix: Proofs}

Before presenting the proof of Theorem 5.1, we state several lemmas. For this, for $z \in \mathbb{Q}^{\ell}$ we define $\Gamma(z)=\{t \in I \mid U(t, z+\omega(t))>U(t, f(t))\}$, and $\Gamma^{n}(z)=\left\{t \in I \mid U^{n}\left(t, z+\omega^{n}(t)\right)>U^{n}\left(t, f^{n}(t)\right)\right\}$ for each $n \in \mathbb{N}$. Let $\mathcal{Z}=\left\{z \in \mathbb{Q}^{\ell} \mid \mu(\Gamma(z))=0\right\}$ and, for each agent $t \in I$, let $\psi(t)=\{z \in$ $\left.\mathbb{R}^{\ell} \mid U(t, z+\omega(t))>U(t, f(t))\right\}$.

Lemma 1. Let $S$ be a coalition of agents blocking the allocation $f$ in the economy $\mathcal{E}_{c}$. Then, for each $i \in\{1, \ldots, \ell+1\}$, there exist $\alpha_{i} \in \mathbb{Q}_{+}, z_{i} \in \mathbb{Q}^{\ell}$, and $t_{i} \in \hat{S}=S \backslash \bigcup_{z \in Z} \Gamma(z)$ such that $\sum_{i=1}^{\ell+1} \alpha_{i}=1, \sum_{i=1}^{\ell+1} \alpha_{i} z_{i}=0$, and $z_{i} \in \psi\left(t_{i}\right)$ 
Proof. As $S$ blocks $f$, there exists $g: S \rightarrow \mathbb{R}_{+}^{\ell}$ such that $\int_{S} g(t) d \mu(t) \leq$ $\int_{S} \omega(t) d \mu(t)$ and $g(t) \succ_{t} f(t)$ for almost all $t \in S$. As $\mu\left(\bigcup_{z \in \mathcal{Z}} \Gamma(z)\right)=0$, $\hat{S}$ is a coalition which also blocks $f$ by the same allocation $g$.

Let $c o(A)$ denote the convex hull of the set $A$. Then $\frac{1}{\mu(\hat{S})} \int_{\hat{S}}(g(t)-$ $\omega(t)) d \mu(t) \in \operatorname{co}((g-\omega)(\hat{S}))$ (see Hüsseinov $(1987,1994))$. Therefore, $0 \in \operatorname{co}\left(\bigcup_{t \in \hat{S}} \psi(t)\right)$. By Caratheodory's theorem, one obtains that there exist $\alpha_{i} \geq 0$, and $z_{i} \in \psi\left(t_{i}\right)$, with $t_{i} \in \hat{S}, i=1, \ldots, \ell+1$, such that $0=\sum_{i=1}^{\ell+1} \alpha_{i} z_{i}$. By continuity and monotonicity of preferences, we can take $\alpha_{i} \in \mathbb{Q}_{+}$and $z_{i} \in \mathbb{Q}^{\ell}$ with $\sum_{i=1}^{\ell+1} \alpha_{i}=1, \sum_{i=1}^{\ell+1} \alpha_{i} z_{i}=0$ and $z_{i} \in \psi\left(t_{i}\right)$.

Lemma 2. For each $\varepsilon>0$ there exists $J_{\varepsilon} \subset I$, with $\mu\left(J_{\varepsilon}\right)>1-\varepsilon$, such that, for each $t \in J_{\varepsilon}, U^{n}(t, \cdot)$ converges to $U(t, \cdot)$ uniformly on compact subsets of $\mathbb{R}_{+}^{\ell}$.

Proof. For $x \in \mathbb{R}_{+}^{\ell}$, by the Lebesgue differentiation theorem, $U^{n}(t, x)$ converges to $U(t, x)$ for almost all $t \in I$. In particular, for each $x \in \mathbb{Q}_{+}^{\ell}$ there exists $J(x) \subset I$, with $\mu(J(x))=1$, such that $U^{n}(t, x)$ converges to $U(t, x)$ for all $t \in J(x)$. Let $J=\bigcap_{x \in \mathbb{Q}_{+}^{\ell}} J(x)$. Then $\mu(J)=1$ and $U^{n}(t, x)$ converges to $U(t, x)$ for all $t \in J$.

On the other hand, by (H.3) $U$ is measurable. Lusin's theorem guarantees that, for each $\varepsilon>0$, there exists a compact set $I_{\epsilon} \subset I$, with $\mu\left(I_{\varepsilon}\right)>1-\varepsilon$, such that $U$ is continuous on $I_{\epsilon}$. Note that $\mu\left(J_{\varepsilon}\right)>1-\varepsilon$, where $J_{\epsilon}=J \bigcap I_{\varepsilon}$.

We show that $U^{n}(t, x)$ converges to $U(t, x)$ for all $t \in J_{\varepsilon}$, for any $x \in \mathbb{R}_{+}^{\ell}$. First, given $x \in \mathbb{R}_{+}^{\ell}$, let $\left(x^{k}\right) \subset \mathbb{Q}_{+}^{\ell}$ be a sequence such that $x^{k} \rightarrow x$. Then, $\lim _{k \rightarrow \infty} U^{n}\left(t, x^{k}\right)=U^{n}(t, x)$ for all $n$ and for all $t \in I$ and $\lim _{n \rightarrow \infty} U^{n}\left(t, x^{k}\right)=$ $U\left(t, x^{k}\right)$ for all $k$ and for all $t \in J_{\varepsilon}$. Moreover, this last convergence is uniform in $k$. In fact, let $K=\left\{\left.U\right|_{J_{\varepsilon}}\left(\cdot, x_{k}\right), k \in \mathbb{N}\right\}$, where $\left.U\right|_{J_{\varepsilon}}$ denotes the restriction of $U$ to $J_{\varepsilon}$. As $U$ is continuous on $J_{\varepsilon}$, we conclude that $K$ is an equicontinuous set. Therefore, applying Ascoli-Arzela's theorem, Moore's lemma and result IV.8.18 in Dunford-Schwartz (1958), we conclude that $U^{n}(t, x)$ converges to $U(t, x)$ for all $t \in J_{\varepsilon}$ and all $x \in \mathbb{R}_{+}^{\ell}$.

Finally, reasoning as before, we see that $\lim _{n \rightarrow \infty} U^{n}\left(t, x^{n}\right)=U(t, x)$ for all $t \in J_{\varepsilon}$. That is, following Carathéodory's notion of continuous convergence (see Royden (1988), Problem 7.40), if $t \in J_{\varepsilon}$, then $U^{n}(t, \cdot)$ converges to $U(t, \cdot)$ uniformly on compact subsets of $\mathbb{R}_{+}^{\ell}$. 
Lemma 3. The function $U^{n}(\cdot, \cdot)$ converges to $U(\cdot, \cdot)$ almost uniformly on I and uniformly on compact subsets of $\mathbb{R}_{+}^{\ell}$. That is, for a compact set $K \subset \mathbb{R}_{+}^{\ell}$, for each $\varepsilon>0$ there exists $J_{\varepsilon} \subset I$, with $\mu\left(J_{\varepsilon}\right)>1-\varepsilon$, such that $U^{n}(\cdot, \cdot)$ converges to $U(\cdot, \cdot)$ uniformly on $J_{\varepsilon} \times K$.

Proof. Let $\varepsilon>0$. By Lemma 2, there exists $J \subset I$, with $\mu(J)>1-\frac{\varepsilon}{2}$, such that, for all $t \in J, U^{n}(t, x)$ converges to $U(t, x)$ for all $x \in \mathbb{R}_{+}^{\ell}$, and this convergence is uniform on $K$. Given $k, m$ positive integers, we define

$$
J_{k, m}=\left\{t \in J|| U^{n}(t, x)-U(t, x) \mid<\frac{1}{m} \text { for all } n \geq k, x \in K\right\}
$$

Then, by the uniform convergence on $K$ for all $t \in J$, we have that $J=$ $\bigcup_{k=1}^{\infty} J_{k, m}$ for all $m$. So, for each $m$, we see that there exists $k(m)$ such that $\mu\left(J \backslash J_{k(m), m}\right)<\varepsilon 2^{-m-1}$. Let $J_{\varepsilon}=\bigcap_{m=1}^{\infty} J_{k(m), m}$. Then, $\mu\left(J \backslash J_{\varepsilon}\right)<\varepsilon$ and therefore, $U^{n}(\cdot, \cdot)$ converges to $U(\cdot, \cdot)$ uniformly on $J_{\varepsilon} \times K$.

Proof of Theorem 5.1. Observe that, if the allocation $x^{n}$ does not belong to $\operatorname{Core}\left(\overline{\mathcal{E}}_{n}\right)$, then the corresponding allocation $f^{n}$ does not belong to $\mathcal{F}^{n}$ $\operatorname{Core}\left(\overline{\mathcal{E}}_{c}^{n}\right)$, and that $\operatorname{Core}\left(\overline{\mathcal{E}}_{c}^{n}\right) \subset \mathcal{F}^{n}$-Core $\left(\overline{\mathcal{E}}_{c}^{n}\right)$. Thus, it is enough to prove the result for $\overline{\mathcal{E}}_{n}$.

For this, suppose that $f \notin \operatorname{Core}\left(\mathcal{E}_{c}\right)$. Then, a coalition $S$ blocks $f$ and, by Lemma 1 , there exist $\alpha_{i} \in \mathbb{Q}_{+}$and $z_{i} \in \psi\left(t_{i}\right), i=1, \ldots, \ell+1$, with $t_{i} \in \hat{S}=S \backslash \bigcup_{z \in Z} \Gamma(z)$ and $\sum_{i=1}^{\ell+1} \alpha_{i}=1$, such that $0=\sum_{i=1}^{\ell+1} \alpha_{i} z_{i}$.

By the definition of $\hat{S}$, as $z_{i} \in \psi\left(t_{i}\right)$, we have that $\mu\left(\Gamma\left(z_{i}\right)\right)>0$. So, there exists $\alpha>0$ such that $\mu\left(\Gamma\left(z_{i}\right)\right) \geq \alpha$ for all $i \in\{1, \ldots, \ell+1\}$. Thus, $z_{i} \in \psi(t)$ for all $t \in \Gamma\left(z_{i}\right)$, Therefore, there exist $B_{i} \subset \Gamma\left(z_{i}\right)$, with $\mu\left(B_{i}\right)<\frac{\alpha}{8}$, and $\delta>0$, such that $U\left(t, z_{i}+\omega(t)\right)-U(t, f(t)) \geq \delta$ for all $t \in \Gamma\left(z_{i}\right) \backslash B_{i}$.

On the other hand, there exist $A \subset I, n_{0}$ and a compact $K \subset \mathbb{R}^{\ell}$ such that $\mu(A)<\frac{\alpha}{8}$ and $f(t), f^{n}(t), z_{i}+\omega^{n}(t) \in K$ for all $t \in A$ and for all $n \geq n_{0}$. By Egoroff's theorem (see Royden (1988)) and Lemma 3, there exists a set of agents $B \subset I$, with $\mu(B)<\frac{\alpha}{8}$, such that $f^{n}(t) \rightarrow f(t)$, $\omega^{n}(t) \rightarrow \omega(t)$ and $U^{n}(t, x) \rightarrow U(t, x)$ uniformly for all $t \notin B$ and for all $x \in K$.

For each $\varepsilon>0$, consider the map $\varphi(\cdot, \varepsilon): I \rightarrow \mathbb{R}_{+}$given by

$$
\varphi(t, \varepsilon)=\sup _{\substack{x \in K \\\|y\| \leq \varepsilon}}|U(t, x+y)-U(t, x)| .
$$

Note that $\varphi(t, \varepsilon)$ converges to 0 when $\varepsilon \rightarrow 0$. Let $I_{\varepsilon}=\left\{t \in I \mid \varphi(t, \varepsilon)<\frac{\delta}{4}\right\}$. As $I=\bigcup_{\varepsilon} I_{\varepsilon}$, there exists $\varepsilon_{0}$ such that $\mu\left(I \backslash I_{\varepsilon_{0}}\right)<\frac{\alpha}{8}$ and $\varphi\left(t, \varepsilon_{0}\right)<\frac{\delta}{4}$ for all $t \in I_{\varepsilon_{0}}$. 
Let $\Gamma^{\prime}\left(z_{i}\right)=\left(\Gamma\left(z_{i}\right) \bigcap I_{\varepsilon_{0}}\right) \backslash\left(A \bigcup B \bigcup B_{i}\right)$. Then $\mu\left(\Gamma^{\prime}\left(z_{i}\right)\right)>\frac{\alpha}{2}$. Moreover, $f^{n}$ (resp., $\omega^{n}$ ) converges to $f$ (resp., to $\omega$ ) uniformly on $\Gamma^{\prime}\left(z_{i}\right)$. So, there exists $n_{1}$ such that $\left\|f^{n}(t)-f(t)\right\|,\left\|\omega^{n}(t)-\omega(t)\right\|<\varepsilon_{0}$ for all $n \geq n_{1}$ and $t \in \Gamma^{\prime}\left(z_{i}\right)$. By the uniform convergence of $U^{n}(t, x)$ with $t \in \Gamma^{\prime}\left(z_{i}\right)$ and $x \in K$, there exists $n_{2}$ such that $\left|U^{n}(t, x)-U(t, x)\right|<\frac{\delta}{4}$ for all $x \in K, t \in \Gamma^{\prime}\left(z_{i}\right)$ and $n \geq n_{2}$. In the same way, there exists $\hat{n}$ such that $\Gamma^{\prime}\left(z_{i}\right) \subset \Gamma^{n}\left(z_{i}\right)$ for all $i \in\{1, \ldots, \ell+1\}$ and $n \geq \hat{n}$. Moreover, $\mu\left(\Gamma^{n}\left(z_{i}\right)\right)>\frac{\alpha}{2}$.

On the other hand, for each $i$ we can write $\alpha_{i}=\frac{\beta_{i}}{\beta}$, with $\beta_{i}, \beta \in \mathbb{N}$ and $\beta_{i} \leq \beta$. By the definition of $\Gamma^{n}\left(z_{i}\right)$, for each $n \geq \hat{n}$ and $i$, there exists a type subset $T_{i}^{n} \subset\left\{1, \ldots, 2^{n}\right\}$ such that $\Gamma^{n}\left(z_{i}\right)=\bigcup_{j \in T_{i}^{n}} I_{j}^{n}$. As $\mu\left(I_{j}^{n}\right)$ converges to zero when $n$ goes to $\infty$, but simultaneously $\mu\left(\Gamma^{n}\left(z_{i}\right)\right)>\frac{\alpha}{2}>0$, we see that there exists $n^{*}$ such that $\operatorname{Card}\left(T_{i}^{n}\right)>\beta$ for all $i$ and $n \geq n^{*}$. Consider $J_{i}^{n} \subset T_{i}^{n}$ with $\operatorname{Card}\left(J_{i}^{n}\right)=\beta_{i}$. Given $n \geq n^{*}$, let $y^{n}$ be an allocation that associates to each agent $j \in J_{i}^{n}$ the consumption vector $y_{j}^{n}=z_{i}+\omega^{n}\left(t_{j, i}\right)$, with $t_{j, i} \in I_{j}^{n} \subset \Gamma^{n}\left(z_{i}\right), 1 \leq j \leq \beta_{i}$. Then, the coalition $J^{n}=\bigcup_{i=1}^{\ell+1} J_{i}^{n}$ blocks the allocation $x^{n}$ for $n \geq n^{*}$ because

$$
\begin{aligned}
& \sum_{i=1}^{\ell+1} \sum_{j=1}^{\beta_{i}}\left(z_{i}+\omega^{n}\left(t_{j, i}\right)\right)=\sum_{i=1}^{\ell+1} \beta_{i} z_{i}+\sum_{i=1}^{\ell+1} \sum_{j=1}^{\beta_{i}} \omega^{n}\left(t_{j, i}\right) \\
& =\beta \sum_{i=1}^{\ell+1} \alpha_{i} z_{i}+\sum_{i=1}^{\ell+1} \sum_{j=1}^{\beta_{i}} \omega^{n}\left(t_{j, i}\right)=\sum_{i=1}^{\ell+1} \sum_{j=1}^{\beta_{i}} \omega^{n}\left(t_{j, i}\right) .
\end{aligned}
$$

Before giving the proof of Theorem 5.2, we present the following two lemmas.

Lemma 4. Let $\left(\succeq_{n}\right) \subset \hat{\mathcal{P}}, \succeq \in \hat{\mathcal{P}}$ and let $U_{n}, U$ in $\hat{\mathcal{U}}$ be the utility functions representing $\succeq_{n}$ and $\succeq$, respectively. Then, $\rho\left(\succeq_{n}, \succeq\right)$ converges to zero iff $U_{n}$ converges to $U$ uniformly on compact subsets of $\mathbb{R}_{+}^{\ell}$.

Proof. For the necessary condition, let $\varepsilon>0, K$ be a compact subset of $\mathbb{R}_{+}^{\ell}$ and $r$ be such that $K \subset B(0, r)=\left\{x \in \mathbb{R}_{+}^{\ell} ;\|x\|<r\right\}$. As $\succeq_{n} \stackrel{\rho}{\rightarrow} \succeq$, for each $\hat{\varepsilon}=\frac{\varepsilon}{1+r^{2}}$, there exists $n_{0}$ such that $\rho\left(\succeq_{n}, \succeq\right) \leq \hat{\varepsilon}$ for all $n \geq n_{0}$. Thus, multiplying and dividing by $1+\|x\|^{2}$, we get $\max _{x \in K}\left|U_{n}(x)-U(x)\right| \leq \varepsilon$ for all $n \geq n_{0}$.

For the sufficient condition, let $\varepsilon>0$. As $U_{n}, U \in \hat{\mathcal{U}}$, there exists a constant $k$, which only depends on $\ell$, such that $U_{n}(x) \leq k\|x\|$ and $U(x) \leq$ $k\|x\|$ for all $n$ and $x \in \mathbb{R}_{+}^{\ell}$. Let $r>1$ be such that $\frac{2 k r}{1+r^{2}}<\varepsilon$. As $U_{n}$ converges to $U$ uniformly on $K=\left\{x \in \mathbb{R}_{+}^{\ell} ;\|x\| \leq r\right\}$, there exists $n_{0}$ such that $\max _{x \in K}\left|U_{n}(x)-U(x)\right| \leq \varepsilon$ for all $n \geq n_{0}$. 
Then, we show that $\max _{x \in K} \frac{\left|U_{n}(x)-U(x)\right|}{1+\|x\|^{2}} \leq \varepsilon$ for all $n \geq n_{0}$. If $x \notin K$, as $\|x\|>r>1$, then

$$
\frac{\left|U_{n}(x)-U(x)\right|}{1+\|x\|^{2}} \leq \frac{\left|U_{n}(x)\right|+|U(x)|}{1+\|x\|^{2}} \leq \frac{2 k\|x\|}{1+\|x\|^{2}} \leq \frac{2 k r}{1+r^{2}} .
$$

This allows us to conclude that $\max _{x \in \mathbb{R}_{+}^{\ell}} \frac{\left|U_{n}(x)-U(x)\right|}{1+\|x\|^{2}} \leq \varepsilon$ for all $n \geq n_{0}$.

Lemma 5. For each agent $t \in I$, let $\succeq_{t}\left(\right.$ resp., $\left.\succeq_{t}^{n}\right)$ be her preference relation in the economy $\mathcal{E}_{c}\left(\right.$ resp..$\left.\overline{\mathcal{E}}_{c}^{n}\right)$, represented by the utility function $U(t, \cdot)$ (resp. $\left.U^{n}(t, \cdot)\right)$. Suppose that $U(t, \cdot) \in \hat{\mathcal{U}}$ for almost all $t \in I$. Then, for every $\varepsilon>0$, there exists $I_{\varepsilon} \subset I$, with $\mu\left(I_{\varepsilon}\right)>1-\varepsilon$, such that $\rho\left(\succeq_{t}^{n}, \succeq_{t}\right)$ converges to zero for every $t \in I_{\varepsilon}$.

Proof. As $U(t, x)=\|x\|$ for all $x$ in the principal diagonal of $\mathbb{R}_{+}^{\ell}$ and for almost all $t \in I$, we see that $U^{n}(t, \cdot) \in \hat{\mathcal{U}}$ for all $t \in I$ and $n$.

On the other hand, by Lemma 2, for every $\varepsilon>0$, there exists $I_{\varepsilon} \subset I$, with $\mu\left(I_{\varepsilon}\right)>1-\varepsilon$, such that $U^{n}(t, \cdot)$ converges to $U(t, \cdot)$ uniformly on compact subsets of $\mathbb{R}_{+}^{\ell}$ for every $t \in I_{\varepsilon}$. Therefore, applying Lemma 4.4, we conclude that $\rho\left(\succeq_{t}^{n}, \succeq_{t}\right)$ converges to zero for every $t \in I_{\varepsilon}$.

Proof of Theorem 5.2. We recall that $f^{n}$ is a feasible allocation in $\overline{\mathcal{E}}_{c}^{n}$ and $f^{n}(t)$ converges to $f(t)$ for almost all $t \in I$. On the other hand, as $\omega^{n}$ converges weakly to $\omega$, the set $\left\{\omega, \omega^{n}: n \in \mathbb{N}\right\}$ is a weakly sequentially compact subset of $L^{1}(I)$. Restricting ourselves to $I_{\varepsilon}$, as in the statement of Lemma 5, with $\varepsilon$ small enough, it is easy to prove that Lemma 5.3 in Kannai (1970) holds. Therefore, the proof of Theorem C in Kannai (1970), on consideration of our restriction to the corresponding $I_{\varepsilon}$, proves our statement.

Proof of Theorem 6.1. Assume that $f \notin \operatorname{Core}\left(\mathcal{E}_{c}\right)$. Then, there exist a coalition $S$ and $g: S \rightarrow \mathbb{R}_{+}^{\ell}$ such that $\int_{S} g(t) d \mu(t) \ll \int_{S} \omega(t) d \mu(t)$, and $U(t, g(t))>U(t, f(t))$ for all $t \in S$. As $\omega^{n}$ converges weakly to $\omega$ in $L^{1}(I)$, there exists $n_{0}$ such that $\int_{S} g(t) d \mu(t) \leq \int_{S} \omega^{n}(t) d \mu(t)$ for all $n \geq n_{0}$. Moreover, by Lusin's theorem we can choose $S$ to be compact and $g$ to be continuous on $S$ (see Hildenbrand (1974), p. 140).

By the continuity properties of $U, f$ and $g$, there exists $\varepsilon>0$ such that, if $\hat{\varepsilon}=(\varepsilon, \ldots, \varepsilon) \in \mathbb{R}_{+}^{\ell}$, then $U(t, g(t))-\frac{\varepsilon}{2}>U(t, f(t)+\hat{\varepsilon})+\frac{\varepsilon}{2}$ for all $t \in S$. Since $f^{n}$ converges to $f$ uniformly on $S$, there exists $n_{1}=n_{1}(\varepsilon)$ such that $f(t)+\hat{\varepsilon} \geq f^{n}(t)$ for all $t \in S$ and $n \geq n_{1}$; and there exists $K$, a compact 
subset of $\mathbb{R}_{+}^{\ell}$, such that $f(S), g(S), f^{n}(S) \subset K$. So, by monotonicity of preferences we see that $U(t, g(t))-\frac{\varepsilon}{2}>U\left(t, f^{n}(t)\right)+\frac{\varepsilon}{2}$ for all $t \in S$.

On the other hand, as $U$ is continuous, there exists $\delta=\delta(\varepsilon, K)$ such that, if $\left|t-t^{\prime}\right|<\delta$ and $x \in K$, then $\left|U\left(t^{\prime}, x\right)-U(t, x)\right|<\frac{\varepsilon}{2}$. Let $n_{2}$ be such that $2^{-n_{2}}<\delta$, and let $\bar{n}=\max \left\{n_{0}, n_{1}, n_{2}\right\}$. Thus, if $n \geq \bar{n}$ and $\left|t-t^{\prime}\right|<\delta$, then $U\left(t^{\prime}, g(t)\right)>U(t, g(t))-\frac{\varepsilon}{2}$ and $U\left(t, f^{n}(t)\right)+\frac{\varepsilon}{2}>U\left(t^{\prime}, f^{n}(t)\right)$.

Note that, if $n \geq \bar{n}$, then $\left|t-t^{\prime}\right|<\delta$ if $t, t^{\prime} \in I_{i}^{n}$, whatever $i$ may be. Therefore, $U\left(t^{\prime}, g(t)\right)>U\left(t^{\prime}, f^{n}(t)\right)$ for all $n \geq \bar{n}$ and $t, t^{\prime} \in I_{i}^{n}$. So, we can conclude that the coalition $S$ blocks $f^{n}$ via $g$ in the economy $\mathcal{E}_{c}^{n}$.

Next we state a lemma which is used in the proof of Theorem 6.2.

Lemma 6. Assume that $\succeq_{t}$ is convex for almost all $t \in I_{i}^{n}$. Let $S \subset I_{i}^{n}$, $\mu(S)>0$. Let $g: S \rightarrow \mathbb{R}_{+}^{\ell}$ be a $\mu$-integrable function and let $x \in \mathbb{R}_{+}^{\ell}$ be such that $g(t) \succ_{i}^{n} x$ for all $t \in S$. Then $\frac{1}{\mu(S)} \int_{S} g(t) d \mu(t) \succ_{i}^{n} x$.

Proof. Let $A=\left\{y \in \mathbb{R}_{+}^{\ell} \mid y \succ_{i}^{n} x\right\}$. First, we show that $A$ is a convex set. For this, let $y_{1}, y_{2} \in A$. Then $y_{1} \succeq_{t} x, y_{2} \succeq_{t} x$ for almost all $t \in I_{i}^{n}$ and there exist $S_{1}, S_{2} \subset I_{i}^{n}$, with $\mu\left(S_{1}\right)>0$ and $\mu\left(S_{2}\right)>0$, such that $y_{1} \succ_{t} x$ for all $t \in S_{1}$ and $y_{2} \succ_{t} x$ for all $t \in S_{2}$. Let $y_{\lambda}=\lambda y_{1}+(1-\lambda) y_{2}$ with $0<\lambda<1$. By convexity of the preferences, $y_{\lambda} \succ_{t} x$ for almost all $t \in S_{1} \bigcup S_{2}$. Thus, $y_{\lambda} \in A$. Therefore $A$ is a convex set. As $g(S) \subset A$ and $\frac{1}{\mu(S)} \int_{S} g(t) d \mu(t) \in \operatorname{co}(g(S))$ (see Hüsseinov $(1987,1994)$ ), we conclude that $\frac{1}{\mu(S)} \int_{S} g(t) d \mu(t) \succ_{i}^{n} x$.

Proof of Theorem 6.2. Suppose that $f \notin \mathcal{F}^{n}$-Core $\left(\hat{\mathcal{E}}_{c}^{n}\right)$ for some $n \geq \bar{n}$. Then, there exist $S \in \mathcal{F}^{n}$ and $g: S \rightarrow \mathbb{R}_{+}^{\ell}$ such that $\int_{S} g(t) d \mu(t) \leq \int_{S} \omega^{n}(t) d \mu(t)$ and $g(t) \succ_{t}^{n} f(t)$ for all $t \in S$. Let $T_{S} \subset\left\{1, \ldots, 2^{n}\right\}$ be such that $S=$ $\bigcup_{i \in T_{S}} I_{i}^{n}$. Then $g(t) \succ_{i}^{n} f_{i}$ for all $t \in I_{i}^{n}$, with $i \in T_{S}$. For each $i \in T_{S}$, let $g_{i}=\frac{1}{\mu\left(I_{i}^{n}\right)} \int_{S} g(t) d \mu(t)$.

Consider $\tilde{g}: S \rightarrow \mathbb{R}_{+}^{\ell}$ given as $\tilde{g}(t)=g_{i}$ if $t \in I_{i}^{n}$. By construction, we see that $\int_{S} \tilde{g}(t) d \mu(t)=\int_{S} g(t) d \mu(t)$. By Lemma $6, g_{i} \succ_{i}^{n} f_{i}$ for all $i \in T_{S}$. By the definition of unanimous preference $\succeq_{i}^{n}$, this is equivalent to the fact that for each $i \in T_{S}$ there exists $S_{i}^{n} \subset I_{i}^{n}$, with $\mu\left(S_{i}^{n}\right)>0$, such that $g_{i} \succeq_{t} f_{i}$ for almost all $t \in I_{i}^{n}$, and $g_{i} \succ_{t} f_{i}$, for all $t \in S_{i}^{n}$. Therefore, the allocation $f$ is blocked by the coalition $S$ in the economy $\mathcal{E}_{c}$. 


\section{References}

Anderson, R.M. (1992): The core in perfectly competitive economies. In: Aumann, R.J., Hart, S. (eds.), Handbook of game theory with economic applications. Vol. I. NorthHolland, Amsterdam, pp. 413-457

Aumann, R.J. (1964): Markets with a continuum of traders. Econometrica 32, 39-50

Aumann, R.J. (1966): Existence of competitive equilibria in markets with a continuum of traders. Econometrica 34, 1-17

Debreu, G. (1954): Representation of a preference ordering by a numerical function. In: Thrall, R.M., Coombs, C.H., Davis, R.L. (eds.), Decision processes. Wiley, New York, pp. 159-165

Debreu, G. (1959): Theory of value: an axiomatic analysis of economic equilibrium. Wiley, New York

Debreu, G., Scarf, H. (1963): A limit theorem on the core of an economy. International Economic Review 4, 235-246

Debreu, G. (1983): Mathematical economics. Twenty papers of Debreu. Cambridge University Press, Cambridge

Dunford, N., Schwartz, J.T. (1958): Linear Operators. I. General theory. Interscience, New York

Edgeworth, F.Y. (1881): Mathematical psychics: an essay on the application of mathematics to the moral sciences. Kegan Paul, London

Eilenberg, S. (1941) Ordered topological spaces. American Journal of Mathematics 63, $39-45$

Hildenbrand, W. (1974): Core and equilibria of a large economy. Princeton University Press, Princeton

Hildenbrand, W. (1982) Core of an economy. In: Arrow, K.J., Intriligator, M.D. (eds.), Handbook of mathematical economics. Vol. II. North-Holland, Amsterdam, pp. 831-877

Hüsseinov, F. [Guseĭnov] (1987): On Jensen's inequality. (Russian) Matematicheskie Zametki 41, 798-806; translated in Mathematical Notes 41, 452-458

Hüsseinov, F. (1994), Interpretation of Aubin's fuzzy coalitions and their extension: relaxation of finite exchange economies. Journal of Mathematical Economics 23, 459-516

Kannai, Y. (1970): Continuity properties of the core of a market. Econometrica 38, 791-815

Kannai, Y. (1972): Continuity properties of the core of a market: a correction. Econometrica 40, 955-958

Mas-Colell, A. (1977): On the continuous representation of preorders. International Economic Review 18, 509-513

Royden, H. (1988): Real analysis. 3rd edition. Macmillan, New York

Wilansky, A. (1983): Topology for analysis. Krieger, Melbourne, FL 